\title{
6d dual conformal symmetry and minimal volumes in AdS
}

\section{Jyotirmoy Bhattacharya and Arthur E. Lipstein}

Centre for Particle Theory \& Department of Mathematical Sciences, Durham University, South Road, Durham DH1 3LE, United Kingdom

E-mail: jyotirmoy.bhattacharya@durham.ac.uk, arthur.lipstein@durham.ac.uk

ABSTRACT: The S-matrix of a theory often exhibits symmetries which are not manifest from the viewpoint of its Lagrangian. For instance, powerful constraints on scattering amplitudes are imposed by the dual conformal symmetry of planar $4 \mathrm{~d} \mathcal{N}=4$ super YangMills theory and the ABJM theory. Motivated by this, we investigate the consequences of dual conformal symmetry in six dimensions, which may provide useful insight into the worldvolume theory of M5-branes (if it enjoys such a symmetry). We find that $6 \mathrm{~d}$ dual conformal symmetry uniquely fixes the integrand of the one-loop 4-point amplitude, and its structure suggests a Lagrangian with more than two derivatives. On integrating out the loop momentum in $6-2 \epsilon$ dimensions, the result is very similar to the corresponding amplitude of $\mathcal{N}=4$ super Yang-Mills theory. We confirm this result holographically by generalizing the Alday-Maldacena solution for a minimal area string in Anti-de Sitter space to a minimal volume M2-brane ending on a pillow-shaped surface in the boundary whose seams correspond to a null-polygon. This involves careful treatment of a prefactor which diverges as $1 / \epsilon$, and we comment on its possible interpretation. We also study 2-loop 4point integrands with $6 \mathrm{~d}$ dual conformal symmetry and speculate on the existence of an all-loop formula for the 4-point amplitude.

KEYwords: AdS-CFT Correspondence, M-Theory, p-branes, Scattering Amplitudes

ARXiv EPrint: 1611.02179 


\section{Contents}

1 Introduction 1

2 6d dual conformal amplitudes $\quad 4$

2.1 Review of dual conformal symmetry 5

2.2 One-loop four-point 6

2.3 Bubbles and triangles 8

2.4 Higher loops 8

3 Minimal volumes in AdS $\quad 9$

$\begin{array}{lll}3.1 \text { General setup } & 10\end{array}$

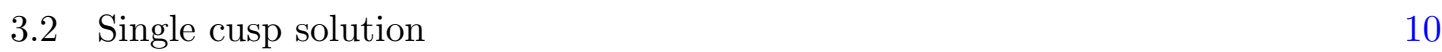

$\begin{array}{lll}3.3 & \text { Four cusp solution } & 11\end{array}$

$\begin{array}{ll}3.4 & \text { Regulated on-shell action } \\ \end{array}$

$\begin{array}{lll}4 & \text { Discussion } & 14\end{array}$

$\begin{array}{ll}\text { A Details of 2-loop 4-point amplitude } & 16\end{array}$

$\begin{array}{ll}\text { B Details of regularized action } & 18\end{array}$

\section{Introduction}

The AdS/CFT conjecture [1] establishes a correspondence between string or M-theory in the near horizon geometry of a stack of D-branes or M-branes to the effective lowenergy world-volume theory of the branes. Although this idea has now grown into a wide framework of gauge-gravity duality with diverse applications, the most prominent and early examples of this conjecture involve maximally supersymmetric field theories on D3, M2 and M5-branes, which are conjectured to be dual to IIB string theory on $\mathrm{AdS}_{5} \times \mathrm{S}^{5}$ [2], M-theory on $\mathrm{AdS}_{4} \times \mathrm{S}^{7}$ [3], and $\mathrm{AdS}_{7} \times \mathrm{S}^{4}$ [4], respectively.

In the first example, the worldvolume theory on D3-branes is $4 \mathrm{~d} \mathcal{N}=4$ super YangMills theory (SYM) [5], which admits an expansion in terms of the 't Hooft parameter, which in turn can also be classified by the topologies of Riemann surfaces [6]. In such an expansion, the topologies with higher genus are suppressed by the rank of the gauge group $N$, and in the limit when $N$ is large, the theory is entirely dominated by planar diagrams. In the planar limit, the theory is believed to be solvable (for a review, see for example [7]).

The second and third examples of the AdS/CFT conjecture are more challenging because M-theory arises as the strong coupling limit of string theory, making it difficult to formulate the worldvolume theories of M-branes. This was however, recently accomplished 
for M2-branes [8]. The key insight was to perform a $\mathbb{Z}_{k}$ orbifold of the space transverse to the branes which breaks $1 / 4$ of the susy when $k>2$, but allows one to define a tunable coupling for the theory. The Lagrangian is a $3 \mathrm{~d}$ superconformal Chern-Simons theory with $\mathcal{N}=6$ susy known as the ABJM theory. ${ }^{1}$ When the orbifold parameter is large, it is dual to IIA string theory on $\mathrm{AdS}_{4} \times \mathrm{CP}^{3}$.

The third example is the least understood because the worldvolume theory describing multiple M5-branes remains elusive. The M5-brane theory is not only crucial for understanding AdS/CFT and M-theory, it also provides the geometric origin of electric-magnetic duality of many supersymmetric theories that arise from dimensional reduction [12-14]. It is believed to be a $6 \mathrm{~d}$ CFT whose field content is a $(2,0)$ tensor multiplet, which consists of a self-dual two-form gauge field, five scalars, and eight fermions. Although the worldvolume theory for a single M5-brane is well-understood [15, 16], it is unclear how to generalize it to more than one. In the absence of a tunable coupling, it is not clear whether such a theory would even admit a Lagrangian description (see [17-24] for some attempts in this direction).

Using modern methods for computing on-shell scattering amplitudes, it is possible to learn a great deal about the S-matrix of many theories without reference to their Lagrangians. Moreover, the S-matrix often exhibits symmetries that are totally obscure from the point of view of the Lagrangian. For example, the planar amplitudes of $\mathcal{N}=4$ SYM [25-27] and the ABJM theory [28-30] exhibit a remarkable property known as dual conformal symmetry in the planar limit. In the context of $\mathcal{N}=4 \mathrm{SYM}$, the dual conformal symmetry can be understood as the ordinary conformal symmetry of a dual Wilson loop whose contour is obtained by arranging the external momenta of the amplitude head to tail [31-36]. Moreover, the amplitude/Wilson loop duality of $4 \mathrm{~d} \mathcal{N}=4 \mathrm{SYM}$ can be derived from the self-duality of IIB string theory under a certain combination of bosonic and fermionic T-duality transformations $[37,38]$. In the case of ABJM theory, the amplitude/Wilson loop duality does not generalize beyond 4-points and the status of the fermionic T-duality in the gravity dual is also unclear [39-41]. Hence, the origin of dual conformal symmetry in the ABJM theory appears to be rather mysterious.

Note that dual conformal symmetry is not equivalent to ordinary superconformal symmetry and when the two are combined, they give rise to infinite dimensional Yangian symmetry for scattering amplitudes [42, 43]. Hence, dual conformal symmetry imposes very powerful constraints on the planar S-matrix. For example, in $\mathcal{N}=4 \mathrm{SYM}$ and ABJM one can use dual conformal symmetry to uniquely fix the 1-loop 4-point integrand, from which the 4-point tree-level amplitudes can be deduced using unitarity methods [44, 45]. The rest of the tree-level S-matrix can then be deduced using BCFW recursion [46], which in principle can be used to deduce the Lagrangian. Hence, apriori if we had no idea how to formulate ABJM or $\mathcal{N}=4 \mathrm{SYM}$, we could have deduced these theories simply by looking for dual conformal invariant S-matrices in $3 \mathrm{~d}$ and $4 \mathrm{~d}$, respectively.

At loop-level, dual conformal symmetry is broken by IR divergences but the four and five points amplitudes of $\mathcal{N}=4 \mathrm{SYM}$ are nevertheless fixed to all orders by an anomalous

\footnotetext{
${ }^{1}$ Prior to the ABJM theory, there were other proposals which have maximal superconformal symmetry, although the interpretation of these theories is not fully understood [9-11].
} 
dual conformal Ward identity [47]. These all-loop formulae, first conjectured by Bern, Dixon, and Smirnov [48], boil down to exponentiating the one-loop amplitude and encoding the coupling dependence through the cusp and collinear anomalous dimensions. This has been confirmed up to four loops in perturbation theory [49-51], as well as at strong coupling using string theory $[31,52,53]$. There is also evidence that a similar all-loop formula exists for the 4-point amplitude of the ABJM theory [54-60]. In particular, the 2-loop 4-pt amplitude of the ABJM theory has an almost identical structure to the 1-loop 4-point amplitude of $\mathcal{N}=4 \mathrm{SYM}$, and the strong coupling calculation in the dual string theory is identical to that of $\mathcal{N}=4 \mathrm{SYM}$ at leading order.

Given the important role played by dual conformal symmetry in the first two examples of the AdS/CFT duality described above, in this note we proceed to explore its implications for the theory of M5-branes, assuming it enjoys such a symmetry. There are of course, several subtleties with this point of view. For instance, it has previously been shown that is not possible to construct a tree-level S-matrix for $(2,0)$ tensor multiplets assuming locality and unitarity [61]. On the other hand, since the $(2,0)$ theory is strongly coupled, it is unclear what the asymptotic states should be. In this note, we will not make any assumptions about the asymptotic states, locality, or even the superconformal symmetry of the theory. Our only assumptions will be that the theory admits something analogous to a planar limit where a semi-classical supergravity description is admissible in the bulk, and that it has rational loop integrands ${ }^{2}$ which exhibit dual conformal symmetry in six dimensions. ${ }^{3}$

In this note, we find that $6 \mathrm{~d}$ dual conformal symmetry fixes the 1-loop 4-point integrand. Like $\mathcal{N}=4 \mathrm{SYM}$, the integrand can be associated with a scalar box diagram with massless external legs, but unlike $\mathcal{N}=4 \mathrm{SYM}$, some of the propagators are squared which suggests an underlying Lagrangian with more than two derivative. We also consider triangle and bubble diagrams with dual conformal integrands but show that they are not important at 4 points. Remarkably, although the 4-point 1-loop integrand that we obtain has a very different spacetime structure than that of $\mathcal{N}=4 \mathrm{SYM}$, its integral is essentially the same (up to scheme-dependent terms). Recalling that a very similar structure was found for the 2-loop 4-point amplitude of the ABJM theory, it therefore appears that 4-point amplitudes with dual conformal symmetry have a universal structure. We also carry out a preliminary analysis of 2-loop 4-point integrands consistent with $6 \mathrm{~d}$ dual conformal symmetry and unitarity. Remarkably, although these integrands have a very different spacetime structure than that of $\mathcal{N}=4 \mathrm{SYM}$, their Mellin-Barnes representation is very similar, suggesting that the similarity to $\mathcal{N}=4 \mathrm{SYM}$ might extend beyond 1-loop.

The all-loop formula for the four-point amplitude of $\mathcal{N}=4$ SYM was first confirmed at strong coupling by Alday and Maldacena who computed the minimal area of a string in AdS whose boundary corresponds to the null polygon obtained by arranging the ex-

\footnotetext{
${ }^{2}$ Since the M5-brane theory is self-dual, it is unclear how to define a topological expansion in terms of an 't Hooft parameter. A similar difficulty occurs for the M2-brane theory, but can be overcome by orbifolding the space transverse to the branes, which introduces a tunable coupling.

${ }^{3}$ Note that $6 \mathrm{~d}(1,1)$ SYM amplitudes have dual conformal symmetry in the four-dimensional sense [62], as do maximal SYM amplitudes in 10d [63] and 3d [64].
} 
ternal momenta of the amplitude head to tail [31]. In fact, this was the first hint of the amplitude/Wilson loop duality. For the M5-brane theory, we would expect the analogous calculation to involve the minimal volume of an M2-brane whose boundary is a $2 \mathrm{~d}$ surface which somehow encodes a null polygon. Remarkably, by relaxing a constraint of the AldayMaldacena solution, we obtain a solution which minimizes the volume of a 2-brane in AdS and the boundary of this solution is a pillow-shaped surface whose seams correspond to a null polygon. ${ }^{4}$ We evaluate the on-shell action for the 2-brane solution and find a structure very similar to the $6 \mathrm{~d}$ 1-loop amplitude, which suggests an amplitude/Wilson surface duality and the existence of an all-loop BDS-like formula for the 4-point amplitude. Unlike the on-shell action of the Alday-Maldacena string solution, the on-shell action of the 2-brane has a prefactor that depends on the dimensional regularization parameter $\epsilon=(6-d) / 2$ and diverges like $1 / \epsilon$ as $\epsilon \rightarrow 0$. This additional divergence is consistent with previous holographic calculations of Wilson surfaces [65, 66], and can be associated with the conformal anomaly of Wilson surfaces [67-70]. ${ }^{5}$

This note is organized as follows. In 2 we explore the consequences of dual conformal symmetry in six dimensions. In particular, we find that dual conformal symmetry fixes the integrand of the 1-loop 4-point amplitude, which we integrate in $d=6-2 \epsilon$ dimensions using the Mellin-Barnes technique. We also initiate the study of 2-loop 4-point amplitudes. In section 3, we find a solution describing a minimal volume 2-brane in AdS whose boundary is a $2 \mathrm{~d}$ surface encoding a null polygon. We then compute the on-shell action for this solution and find that it has a very similar structure to the 1-loop 4-point amplitude we computed in section 2, suggesting the existence of an amplitude/Wilson surface duality and an all-loop formula for the 4-point amplitude analogous to the BDS formula of $\mathcal{N}=4 \mathrm{SYM}$. Finally, in section 4 we present our conclusions and describe future directions. Appendix A gives more details about 2-loop 4-point integrands and appendix B provides more details about the calculation of the on-shell action of the 2-brane in AdS.

\section{$26 d$ dual conformal amplitudes}

Let us consider a hypothetical 6d theory for which a planar limit can be defined and whose loop integrands are rational. We shall study the consequences of dual conformal symmetry for such a theory, primarily focusing on the 4-point case. We shall also assume that in the planar limit it is possible to define color-ordered amplitudes which are cyclically symmetric (the definition of color-ordering in gauge theories can be found in many reviews, see for example [73]). In $\mathcal{N}=4 \mathrm{SYM}$ and the ABJM theory, it is possible to encode the four-point amplitudes with all possible asymptotic states into a single quantity known as a superamplitude. When this quantity is divided by the tree-level four-point superamplitude, the resulting function does not depend on asymptotic states and is determined to all orders by dual conformal symmetry in the planar limit. Moreover, it can be computed at strong

\footnotetext{
${ }^{4}$ Note that since the original null polygon of [31] is embedded within this more general surface, it is parameterized by the same two parameters corresponding to the Mandelstam variables $s$ and $t$ in the amplitude picture.

${ }^{5}$ Also see $[71,72]$ for related discussion of Wilson surface operators in the M5-brane theory.
} 


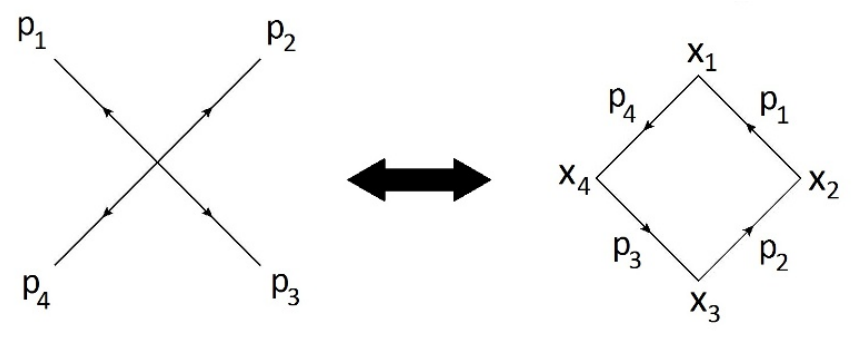

Figure 1. Arranging the external momenta of a scattering head-to-tail gives a null polygon whose vertices are coordinates of the dual space.

coupling using holographic methods. Our goal in the present paper is to deduce such a function in six dimensions, which only relies on the assumption of dual conformal symmetry and does not require knowing the asymptotic states. The four-point superamplitude can then in principle be obtained by multiplying this quantity by the tree-level four-point superamplitude, whose structure is an important open question. We shall therefore define the quantity $\mathcal{M}_{n}=\mathcal{A}_{n} / \mathcal{A}_{n}^{\text {tree }}$. This quantity can then be expanded in powers of the 't Hooft coupling $\lambda$ :

$$
\mathcal{M}_{n}=1+\sum_{l=1}^{\infty} \lambda^{l} \mathcal{M}_{n}^{(l)} .
$$

In the next subsections we will review the concept of dual conformal symmetry, use it to deduce the 1-loop 4-point contribution $\mathcal{M}_{4}^{(1)}$, and analyze the possible structure of $\mathcal{M}_{4}^{(2)}$.

\subsection{Review of dual conformal symmetry}

Dual conformal symmetry can be seen by arranging the external momenta of an amplitude into a polygon and writing the amplitude as a function of the vertices of this polygon as shown in figure 1. Note that this relies on the ability to cyclically order the external particles and is therefore only well-defined in the planar limit. In equations, the coordinates of the dual space are defined by

$$
x_{i}^{\mu}-x_{i+1}^{\mu}=p_{i}^{\mu}
$$

where $p_{i}^{\mu}$ is the momentum of the $i$ th leg. These coordinates automatically incorporate momentum conservation. Dual superconformal symmetry then corresponds to conformal symmetry in the dual space. Note that the amplitudes are manifestly invariant under dual translations since they depend on external momenta which correspond to differences of points in the dual space. Dual conformal symmetry implies the nontrivial property that they transform covariantly under inversions in the dual space:

$$
x_{i}^{\mu} \rightarrow \frac{x_{i}^{\mu}}{x_{i}^{2}}
$$

Note that dual conformal symmetry is not equivalent to ordinary conformal symmetry and imposes very powerful constraints on scattering amplitudes. For example, in $\mathcal{N}=4$ SYM, although this symmetry is broken by IR divergences, it nevertheless determines the 


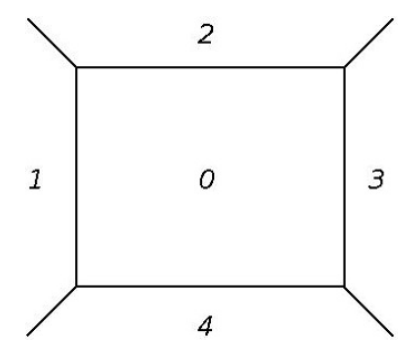

Figure 2. One loop box.

finite part of the four and five-point amplitudes to all orders in the t-Hooft coupling via an anomalous dual conformal Ward identy. Beyond five points it is possible to introduce a function of dual conformal cross ratios and still satisfy the anomalous dual conformal Ward identity, so the amplitudes are given by the BDS formula times a nontrivial remainder function.

\subsection{One-loop four-point}

In $6 \mathrm{~d}$, dual conformal symmetry and cyclic invariance fix the one-loop four point integrand to have the following form:

$$
\mathcal{M}_{4}^{(1)}=\frac{1}{8} \int \frac{d^{D} x_{0} x_{13}^{2} x_{24}^{2}}{x_{10}^{2} x_{20}^{2} x_{30}^{2} x_{40}^{2}}\left(\frac{x_{13}^{2}}{x_{01}^{2} x_{03}^{2}}+\frac{x_{24}^{2}}{x_{02}^{2} x_{04}^{2}}\right) .
$$

where $x_{i j}=x_{i}-x_{j}$ and the factor of $\frac{1}{8}$ is a convention tied to our definition of the coupling $\lambda$ in (2.1). This can be represented using a 1-loop box diagram depicted in figure 2, where each edge of the box corresponds to a propagator in the integrand. It is also possible to define dual conformal triangle and bubble integrals, but as we describe in the next subsection, the former do not contribute at 4-points and the latter evaluate to a constant and woudl therefore only modify scheme-dependent terms in the 4-point 1-loop amplitude. Note that the integrand in (2.3) is manifestly invariant under translations in the dual space. It is also easy to see that when $D=6$, it is invariant under dual inversions, under which the measure and propagators transform as follows:

$$
d x_{0}^{D} \rightarrow \frac{d x_{0}^{D}}{\left(x_{0}^{2}\right)^{D}}, \quad x_{i j}^{2} \rightarrow \frac{x_{i j}^{2}}{x_{i}^{2} x_{j}^{2}} .
$$

Since dual translations and inversions are sufficient to generate the dual conformal group, this establishes dual conformal symmetry of the integrand when $D=6$. An unusual feature of the integrand is that two of the propagators in each term are squared, which suggests that if there is an underlying Lagrangian, it may correspond to a higher derivative theory. It is worth noting that four-derivative terms appear in certain non-unitary $6 \mathrm{~d}$ superconformal theories [74, 75], so it would interesting to study the scattering amplitudes of these theories. Another possibility is that the underlying theory is unitary but non-local. We will return to these points in section 4 . 


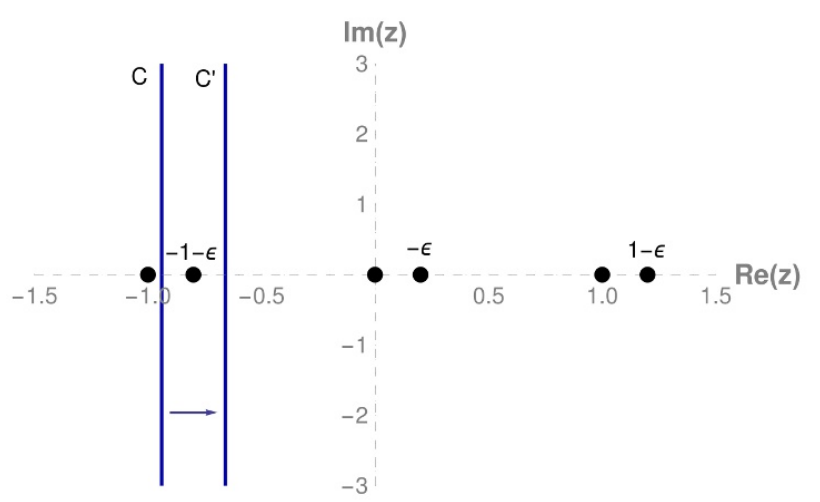

Figure 3. The contour for the integral in (2.4).

The integral in (2.3) is IR divergent, so we will regulate it by taking $D=6-2 \epsilon$, where $\epsilon<0$. Although this breaks dual conformal symmetry, it does so in a controlled and well-understood way as we will see shortly.

This integral can then be evaluated using Mellin-Barnes techniques, as described in [76]. In general, an integral of this form has the following Mellin-Barnes representation:

$$
\begin{aligned}
\int \frac{d^{D} x_{0}}{\Pi_{i=1}^{4}\left(x_{0 i}^{2}\right)^{a_{i}}}= & \frac{(-1)^{a} i \pi^{D / 2}}{\Gamma(D-a) \Pi_{i=1}^{4} \Gamma\left(a_{i}\right) t^{a-D / 2}} \int_{c-i \infty}^{c+i \infty} \frac{d z}{2 \pi i}\left(\frac{s}{t}\right)^{z} \Gamma(-z) \Gamma(a-D / 2+z) \\
& \times \Gamma\left(a_{1}+z\right) \Gamma\left(a_{3}+z\right) \Gamma\left(D / 2-a_{1}-a_{3}-a_{4}-z\right) \Gamma\left(D / 2-a_{1}-a_{2}-a_{3}-z\right),
\end{aligned}
$$

where $s=x_{13}^{2}, t=x_{24}^{2}, a=\sum_{i=1}^{4} a_{i}$, and $c$ is a complex number chosen such that the arguments of the gamma functions have positive real part. For the integral in (2.3) with $D=6-2 \epsilon$, we obtain

$$
\mathcal{M}_{4}^{(1)}=\frac{s t^{-1-\epsilon}}{8 \Gamma(-2 \epsilon)} \int_{c-i \infty}^{c+i \infty} \frac{d z}{2 \pi i}\left(\frac{s}{t}\right)^{z} \Gamma(-z) \Gamma(3+\epsilon+z) \Gamma(1+z)^{2} \Gamma(-1-\epsilon-z)^{2}+s \leftrightarrow t .
$$

Since $\epsilon<0$, one must choose $-1<\Re(c)<-1-\epsilon$ so that the arguments of the gamma functions have positive real part. The integral generates poles in $\epsilon$ which arise from the terms $\Gamma(1+z)^{2} \Gamma(-1-\epsilon-z)^{2}$. If we shift the contour to the right while picking up the residue at $z=-1-\epsilon$ as depicted in figure 3, the integral over the new contour does not have poles in $\epsilon$ and is actually $\mathcal{O}(\epsilon)$ due to the prefactor of $1 / \Gamma(-2 \epsilon)$. Hence, to $\mathcal{O}(\epsilon)$ the amplitude is given by the residue at $z=-1-\epsilon$ :

$$
e^{\epsilon\left(\gamma_{E}-2\right)} \mathcal{M}_{4}^{(1)}=-\frac{1}{2 \epsilon^{2}}\left(\left(\frac{\mu^{2}}{s}\right)^{\epsilon}+\left(\frac{\mu^{2}}{t}\right)^{\epsilon}\right)+\frac{1}{4} \ln ^{2} \frac{s}{t}+\frac{\pi^{2}}{3}+\frac{1}{8}
$$

where $\gamma_{E}$ is the Euler-Mascheroni constant and $\mu^{2}$ is the renormalization scale.

Note that our result for the 1-loop 4-point amplitude six dimensions is the same as the 1-loop amplitude of $\mathcal{N}=4 \mathrm{SYM}$, except for the constant term. Had we multiplied by $e^{\epsilon \gamma}$ than $e^{\epsilon\left(\gamma_{E}-2\right)}$, this would give rise to $\mathcal{O}\left(\frac{1}{\epsilon}\right)$ terms and would modify the constant term, which reflects that these terms are scheme-dependent. In section 3 , we will obtain 


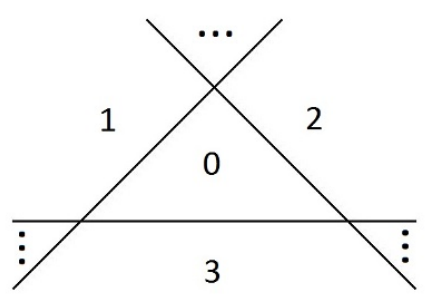

Figure 4. Triangle diagrams.

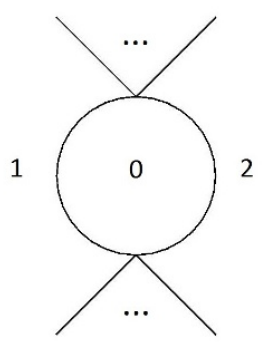

Figure 5. Bubble diagrams.

the same structure at strong coupling by computing the minimal volume of a 2-brane in AdS, which suggests that an anomalous dual conformal Ward identity fixes the finite part of the 4-point amplitude to all orders as it does in $\mathcal{N}=4 \mathrm{SYM}$.

\subsection{Bubbles and triangles}

In the previous section, we focused on 1-loop box diagrams. In principle, one can also consider triangle or bubble diagrams, as depicted in figures 4 and 5. Dual conformal symmetry restricts triangle integrals to have the form

$$
\mathcal{M}_{\text {triangle }}=\int d^{6} x_{0} \frac{\left(x_{12}^{2}\right)^{\alpha}\left(x_{23}^{2}\right)^{\beta}\left(x_{31}^{2}\right)^{\gamma}}{\left(x_{01}^{2}\right)^{\nu_{1}}\left(x_{02}^{2}\right)^{\nu_{2}}\left(x_{03}^{2}\right)^{\nu_{3}}}
$$

where $\nu_{1}+\nu_{2}+\nu_{3}=6$ and $(\alpha, \beta, \gamma)=\frac{1}{2}\left(\nu_{1}+\nu_{2}-\nu_{3},-\nu_{1}+\nu_{2}+\nu_{3}, \nu_{1}-\nu_{2}+\nu_{3}\right)$. From this equation, we see that such contributions only exist for $n>4$ legs (for $n=4$, they collapse to bubble integrals which we will describe shortly). Furthermore, using the results of [77], one finds that they evaluate to rational functions of the kinematic invariants:

$$
\mathcal{M}_{\text {triangle }}=\pi^{n / 2} i^{1-n}\left(x_{12}^{2}\right)^{\alpha+\nu_{3}-3}\left(x_{23}^{2}\right)^{\beta+\nu_{1}-3}\left(x_{31}^{2}\right)^{\gamma+\nu_{2}-3} \Pi_{i=1}^{3} \frac{\Gamma\left(3-\nu_{i}\right)}{\Gamma\left(\nu_{i}\right)} .
$$

For any number of legs, one can define the following dual conformal 1-loop bubble integrals:

$$
\mathcal{M}_{\text {bubble }}=\int d^{6} x_{0}\left(\frac{x_{i j}^{2}}{x_{0 i}^{2} x_{0 j}^{2}}\right)^{3} .
$$

Note that this integral must be a function of the kinematic invariant $x_{i j}^{2}$ but has mass dimension zero. Dimensional analysis therefore implies that it must evaluate to a constant, which can be explicitly verified using the formulae in appendix A of [76]. Hence, a bubble contribution would modify the constant term of the 1-loop 4-point amplitude in (2.5), but as we explained in the previous section, the constant term is scheme-dependent in any case.

\subsection{Higher loops}

In this section we will consider 2-loop 4-point amplitudes with dual conformal symmetry in six dimensions. In $\mathcal{N}=4 \mathrm{SYM}$, the 4-point 2-loop integrand has a double box topology, as depicted in figure 6. To simplify the analysis we will focus on double box diagrams, 


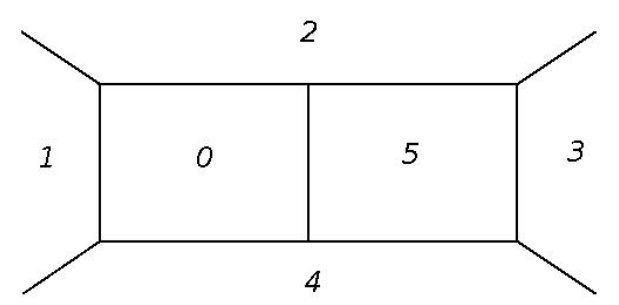

Figure 6. Two loop box.

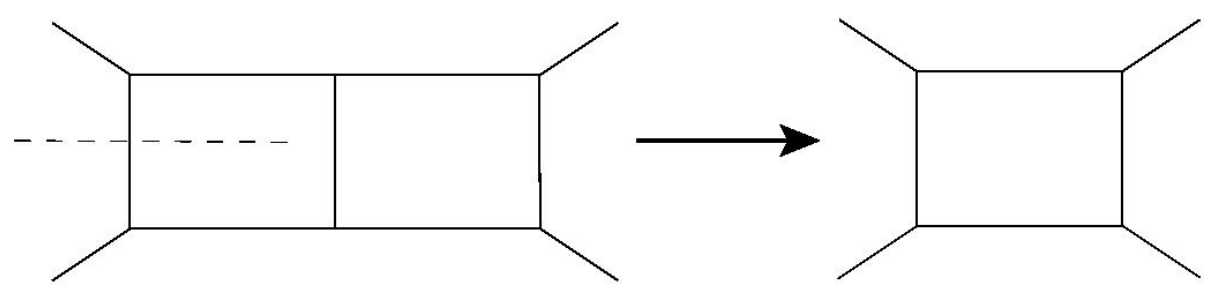

Figure 7. Cutting a propagator of the 2-loop box gives a 1-loop box.

although contributions with triangle or bubble subdiagrams deserve further study. In this case, there are ten possible structures consistent with dual conformal symmetry in $D=6$, two of which reduce to the 1-loop integrand in (2.3) after cutting the propagators with momentum $x_{01}$ or $x_{53}$, as depicted in figure 7 . These two integrands are given by

$$
\begin{aligned}
& \int d^{D} x_{0} d^{D} x_{5} \frac{\left(x_{13}^{2}\right)^{2}\left(x_{24}^{2}\right)^{2}}{\left(x_{01}^{2}\right)^{2} x_{02}^{2} x_{04}^{2} x_{52}^{2}\left(x_{53}^{2}\right)^{2} x_{54}^{2}\left(x_{05}^{2}\right)^{2}}+s \leftrightarrow t \\
& \int d^{D} x_{0} d^{D} x_{5} \frac{x_{13}^{2}\left(x_{24}^{2}\right)^{4}}{x_{01}^{2}\left(x_{02}^{2}\right)^{2}\left(x_{04}^{2}\right)^{2}\left(x_{52}^{2}\right)^{2} x_{53}^{2}\left(x_{54}^{2}\right)^{2} x_{05}^{2}}+s \leftrightarrow t .
\end{aligned}
$$

For example, if we cut the propagator with momentum $x_{01}$ (which amounts to taking this momentum on-shell), then the momenta $x_{02}$ and $x_{04}$ become on-shell and we can re-label $x_{05}$ as $x_{15}$ since $x_{0}$ is no longer integrated over. Discarding on-shell momenta, the two integrands above reduce to terms appearing in the 1-loop integrand (2.3).

In appendix A, we list all ten integrands consistent with dual conformal symmetry as well as their Mellin-Barnes representations. The Mellin-Barnes representation of the two distinguished integrals in (2.6) are remarkably similar to that of $\mathcal{N}=4 \mathrm{SYM}$, even though their spacetime representation looks completely different. This suggests that the 2-loop 4-point amplitude of a $6 \mathrm{~d}$ dual conformal theory should be very closely related to that of $\mathcal{N}=4 \mathrm{SYM}$, as we found at 1-loop. It would be very interesting to integrate these expressions in $D=6-2 \epsilon$ dimensions and see if they arise from exponentiating the 1-loop amplitude, which would suggest a BDS-like formula.

\section{Minimal volumes in AdS}

At strong coupling, the duality between $\mathcal{N}=4$ super-Yang-Mills and string theory on $\mathrm{AdS}_{5} \times S_{5}$ was exploited in [31] to verify the validity of the BDS form of the four point 
amplitude. This was performed by finding an extremal surface $\Sigma$ in $\mathrm{AdS}_{5}$, which ends on a four-sided null-polygon at the boundary. This boundary polygon can be thought of as the contour of a Wilson-loop dual to the four point amplitude. The extremal surface $\Sigma$ then constitutes the world-sheet of the probe-string ending on the boundary Wilsonloop. The extremality of $\Sigma$ implies that the string world-sheet satisfies the equations of motion following from Nambu-Goto action. The four point amplitude is then obtained by evaluating the on-shell action for the string world-sheet and exponentiating.

In this section we will find a simple generalization of the Alday-Maldacena solution that minimizes the action for a 2-brane in $\mathrm{AdS}_{d}$, with $d \geq 5$. Note that $11 \mathrm{~d}$ supergravity admits a solution of $\mathrm{AdS}_{7} \times S^{4}$ with the 3 -form potential having non-zero legs only along the sphere, which describes a stack of M5-branes in the supergravity limit. Hence, our solution can then be interpreted as a single $M 2$-brane in the near horizon geometry of a stack of M5-branes (since the solution is localized on the sphere, the 3-form can be neglected) $[78,79]$.

\subsection{General setup}

The action for an M2 brane is given by the induced volume on the brane

$$
\mathcal{S}=\int d^{3} \xi \mathcal{L}=\mathcal{T}_{M 2} \int d^{3} \xi \sqrt{\operatorname{Det}\left(G_{\mu \nu} \frac{\partial X^{\mu}}{\partial \xi^{a}} \frac{\partial X^{\nu}}{\partial \xi^{b}}\right)},
$$

where $\mathcal{T}_{M 2}$ is the M2-brane tension, $\left(\xi_{1}, \xi_{2}, \xi_{3}\right)$ are the worldvolume coordinates of the brane, and $X^{\mu}$ are the coordinates of the target space with metric $G_{\mu \nu}$. We will take the target space to be $\mathrm{AdS}_{d}$ (with $d \geq 5$ ). In the Poincare patch the metric is

$$
G_{\mu \nu} d X^{\mu} d X^{\nu}=\frac{-d y_{0}^{2}+r^{2}+\Sigma y_{i}^{2}}{r^{2}}
$$

For the rest of the discussion we will set

$$
y_{i}=0, \forall i=4,5, \ldots
$$

and focus on a $\mathrm{AdS}_{5}$ slice of (3.2). This $\mathrm{AdS}_{5}$ can be embedded in $\mathbb{R}^{2,4}$ by the relation

$$
-Y_{-1}^{2}-Y_{0}^{2}+Y_{1}^{2}+Y_{2}^{2}+Y_{3}^{2}+Y_{4}^{2}=-1 .
$$

These coordinates are related to the Poincare coordinates by

$$
Y^{\mu}=\frac{y^{\mu}}{r}, \text { where } \mu=0,1,2,3, \quad Y_{-1}+Y_{4}=\frac{1}{r}, \quad Y_{-1}-Y_{4}=\frac{r^{2}+y^{\mu} y_{\mu}}{r} .
$$

\subsection{Single cusp solution}

In terms of embedding coordinates, the single cusp solution for a 2-brane satisfies the following constraints:

$$
Y_{3}=0, \quad Y_{0}^{2}-Y_{-1}^{2}=Y_{1}^{2}-Y_{4}^{2} .
$$

This is essentially the same as the single-cusp Alday-Maldacena solution with one constraint $\left(Y_{2}=0\right)$ removed, which can be motivated by noting that a 2 -brane is one dimension higher 
than a string and therefore requires one less condition to specify it. Nevertheless, it is rather nontrivial that the constraints in (3.6) actually describe a 2-brane solution. Indeed, when the constraints are transformed to Poincare coordinates using (3.5), they have a very different structure than the Alday-Maldacena solution:

$$
r=\sqrt{2\left(y_{0}^{2}-y_{1}^{2}\right)-y_{2}^{2}}, \quad y_{3}=0 .
$$

If we use the worldvolume diffeomorphsim symmetry to set $\left(\xi_{1}, \xi_{2}, \xi_{3}\right)=\left(y_{0}, y_{1}, y_{2}\right)$, we find that (3.7) is indeed a solution to the Nambu-Goto equations of motion for a 2-brane in AdS. After realizing how to lift the single cusp solution for a string to that of a 2-brane, it is straightforward to generate a four-cusp solution for a 2-brane following the procedure in [31], which we shall describe in the next section.

\subsection{Four cusp solution}

On performing a set of $\mathrm{SO}(2,4)$ transformations following [31], the constraints in (3.6) become

$$
Y_{4}=0, \quad Y_{0} Y_{-1}=Y_{1} Y_{2}
$$

In terms of Poincare coordinates (3.8) translates to

$$
y_{0}=y_{1} y_{2}, \quad r=\sqrt{\left(1-y_{1}^{2}\right)\left(1-y_{2}^{2}\right)-y_{3}^{2}},
$$

where we have made use of (3.5). Choosing $\left(\xi_{1}, \xi_{2}, \xi_{3}\right)=\left(y_{0}, y_{1}, y_{2}\right)$ gives a 4-cusp solution to the equations of motion following from (3.1). Note that this solution describes a minimalvolume 2-brane in AdS and can be obtained from the minimal-area string solution in [31] by writing it in embedding coordinates and relaxing a constraint $\left(Y_{3}=0\right)$. In fact, it can be generalized to an infinite family of $p$-brane solutions in AdS:

$$
y_{0}=y_{1} y_{2}, \quad r=\sqrt{\left(1-y_{1}^{2}\right)\left(1-y_{2}^{2}\right)-\sum_{i=3}^{p+1} y_{i}^{2}}
$$

These solutions have the remarkable property that their on-shell actions factorize into the on-shell action of the minimal string solution times a contribution from the remaining $p-2$ directions of the world-volume, although this factorization does not occur at the level of the classical solutions themselves.

Note that the $r \rightarrow 0$ limit of this solution is a two dimensional surface on the boundary of AdS which may be taken as the contour of a Wilson surface in the $6 \mathrm{~d}$ dual field theory. In figure 8 , we have plotted this surface by projecting it on the spaces orthogonal to $y_{0}$ and $y_{3}$, respectively. From this figure, we see that the Wilson surface can be visualized as a pillow with four seams corresponding to the edges of a null polygon. Moreover, if we identify the edges with the momenta of a scattering amplitude, the resulting Mandelstam variables satisfy $s=t$. 

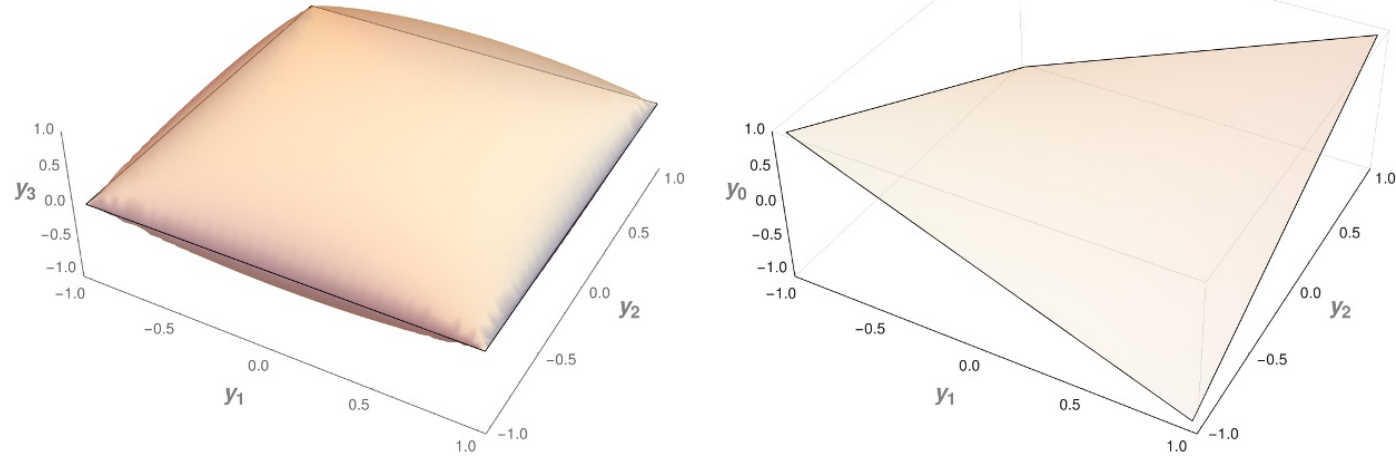

Figure 8. Plots of the boundary surface (3.12), obtained by projecting it on the space orthogonal to $y_{0}$, and $y_{3}$ respectively, for $b=0(s=t)$. When projected orthogonal to $y_{3}$, the surface reduces to a null polygon with its interior filled.

To generalize this solution to $s \neq t$ we can simply apply a boost to (3.8) followed by a rescaling following [31] to obtain

$$
\begin{aligned}
y_{0} & =\frac{a \sqrt{1+b^{2}} \sinh \xi_{1} \sinh \xi_{2}}{\cosh \xi_{1} \cosh \xi_{2}+b \sinh \xi_{1} \sinh \xi_{2}}, \quad y_{1}=\frac{a \sinh \xi_{1} \cosh \xi_{2}}{\cosh \xi_{1} \cosh \xi_{2}+b \sinh \xi_{1} \sinh \xi_{2}} \\
y_{2} & =\frac{a \cosh \xi_{1} \sinh \xi_{2}}{\cosh \xi_{1} \cosh \xi_{2}+b \sinh \xi_{1} \sinh \xi_{2}}, \quad y_{3}=\frac{a \xi_{3}}{\cosh \xi_{1} \cosh \xi_{2}+b \sinh \xi_{1} \sinh \xi_{2}} \\
r & =\frac{a \sqrt{1-\xi_{3}^{2}}}{\cosh \xi_{1} \cosh \xi_{2}+b \sinh \xi_{1} \sinh \xi_{2}}
\end{aligned}
$$

where the world sheet coordinates $\left\{\xi_{1}, \xi_{2}, \xi_{3}\right\}$, must take values in $[-1,1]$ and we make the following identification of the Mandelstam variables with the parameters $a, b$ of the solution:

$$
-s=\frac{8 a^{2}}{(2 \pi)^{2}(1-b)^{2}}, \quad-t=\frac{8 a^{2}}{(2 \pi)^{2}(1+b)^{2}} .
$$

It is not difficult to check that (3.10) solves the equations of motion arising from (3.1) and reduces to (3.9) when $b=0$, which corresponds to $s=t$. Also note that if we replace $\xi_{3}$ by an arbitrary function of $f\left(\xi_{3}\right) \in[-1,1]$ in $(3.10)$, it remains a valid solution of the equations of motion. This is simply related to world-sheet diffeomorphism invariance.

Taking $r \rightarrow 0$ gives the following equations for the surface in boundary:

$$
\begin{aligned}
& y_{0}=\frac{a \sqrt{1+b^{2}} \sinh \xi_{1} \sinh \xi_{2}}{\cosh \xi_{1} \cosh \xi_{2}+b \sinh \xi_{1} \sinh \xi_{2}}, \quad y_{1}=\frac{a \sinh \xi_{1} \cosh \xi_{2}}{\cosh \xi_{1} \cosh \xi_{2}+b \sinh \xi_{1} \sinh \xi_{2}}, \\
& y_{2}=\frac{a \cosh \xi_{1} \sinh \xi_{2}}{\cosh \xi_{1} \cosh \xi_{2}+b \sinh \xi_{1} \sinh \xi_{2}}, \quad y_{3}=\frac{a}{\cosh \xi_{1} \cosh \xi_{2}+b \sinh \xi_{1} \sinh \xi_{2}} .
\end{aligned}
$$

Although these equations are identical to the 4-cusp string solution of Alday-Maldacena solution [31], they are now in Minkowski space rather than AdS, which makes the two surfaces distinct. 
On plugging the solution (3.10) back into Lagrangian (3.1) and restoring the AdS radius $R$, we get

$$
\mathcal{L}=\frac{R^{3} \mathcal{T}_{M 2}}{\left(1-\xi_{3}^{2}\right)^{2}}
$$

which is independent of the coordinates $\xi_{1}$ and $\xi_{2}$. This is not surprising, since $\xi_{1}, \xi_{2}$ are the corresponding coordinates of the string world-sheet in [31], where the Lagrangian simply evaluated to unity on substituting the full solution. In the next section, we will describe how to evaluate the on-shell action, which is divergent and therefore needs to be regulated.

\subsection{Regulated on-shell action}

To implement the analogue of dimensional regularization in the bulk, we follow the method outlined in [31] and define the regularized action as

$$
\mathcal{S}_{\text {reg }}=\int \frac{\mathcal{L}_{\epsilon=0}}{r^{\epsilon}}
$$

where $\epsilon<0$. In [31], this regularization was motivated by analytically continuing the near-horizon metric of black D-banes. Since we are computing the minimal volume of an M2-brane in the bulk, it would be interesting to first dimensionally reduce $\mathrm{AdS}_{7}$ on a circle and then apply the above procedure to compute the on-shell action of the dimensionally reduced solution. In this paper, we will stick with regularization in (3.14) for simplicity.

Although it is difficult to obtain a 4-cusp solution for the regularized action in (3.14), it suffices to know the single-cusp solution for the regularized action. In particular, the single-cusp solution in (3.7) generalizes to

$$
r\left(y_{0}, y_{1}, y_{2}\right)=\sqrt{(2+\epsilon)\left(y_{0}^{2}-y_{1}^{2}\right)-y_{2}^{2}}, \quad y_{3}\left(y_{0}, y_{1}, y_{2}\right)=0
$$

as a solution of the regularized action (3.14). Now we wish to modify the solution in (3.10), so that it reduces to the regulated solution (3.15) near the cusps. This can be accomplished by the following redefinitions:

$$
r \rightarrow \sqrt{1+\frac{\epsilon}{2}} r_{\epsilon=0}, y_{3} \rightarrow \sqrt{1+\frac{\epsilon}{2}}\left(y_{3}\right)_{\epsilon=0}, y_{i} \rightarrow\left(y_{i}\right)_{\epsilon=0}, \text { for } i=0,1,2
$$

where the $\epsilon=0$ solution refers to that in (3.10).

Upon substituting the solution in (3.16) into the regulated action (3.14), we find that the integral over $\xi_{3}$ decouples from the $\xi_{1}, \xi_{2}$ integrals and we obtain

$$
i \mathcal{S}_{\text {reg }}=\mathcal{T}_{\epsilon} \tilde{\mathcal{S}}
$$

where

$$
\mathcal{T}_{\epsilon}=R^{3} \mathcal{T}_{M 2} \int \frac{d \xi_{3}}{\left(1-\xi_{3}^{2}\right)^{2+\frac{\epsilon}{2}}}=-\frac{R^{3} \mathcal{T}_{M_{2}}}{\epsilon}+\mathcal{O}\left(\epsilon^{0}\right)
$$


and $^{6}$

$$
\tilde{\mathcal{S}}=-\frac{\pi \Gamma\left(-\frac{\epsilon}{2}\right)^{2}}{\Gamma\left(\frac{1-\epsilon}{2}\right)^{2}}\left[{ }_{2} F_{1}\left(\frac{1}{2},-\frac{\epsilon}{2}, \frac{1-\epsilon}{2} ; b^{2}\right)+\frac{3}{2}\right]+\mathcal{O}(\epsilon) .
$$

Recalling that $\mathcal{T}_{M_{2}}=\frac{1}{(2 \pi)^{2} l_{p}^{3}}$ and that in the near-horizon limit of a stack of $N$ M5-branes $\left(\frac{R}{l_{p}}\right)^{3}=\pi N$, we finally obtain

$$
i \mathcal{S}_{\mathrm{reg}}=-\frac{N}{4 \pi \epsilon}\left[2 \mathcal{S}_{\mathrm{div}, s}+2 \mathcal{S}_{\mathrm{div}, t}+\frac{1}{4} \ln ^{2}\left(\frac{s}{t}\right)+C\right],
$$

where $\mu$ is the renormalization scale,

$$
\mathcal{S}_{\mathrm{div}, s}=-\frac{1}{\epsilon^{2}}\left(\frac{\mu^{2}}{s}\right)^{\epsilon / 2}+\frac{2 \ln 2}{\epsilon}\left(\frac{\mu^{2}}{s}\right)^{\epsilon / 2}
$$

(with a similar formula for $\mathcal{S}_{\operatorname{div}, t}$ ), and

$$
C=\frac{\pi^{2}}{3}-8 \ln ^{2} 2-\frac{3}{2}
$$

Note that the $\mathcal{O}\left(\frac{1}{\epsilon}\right)$ terms in $\mathcal{S}_{\text {div }}$ and the constant term $C$ are scheme-dependent since they can be altered by rescaling $\mu$.

In terms of the on-shell action for the minimal 2-brane in (3.18), the expectation value of the Wilson surface with contour in (3.12) is given by

$$
\mathcal{M}_{4}=e^{i \mathcal{S}_{\text {reg }}} .
$$

Note that the on-shell action has a very similar structure to the 1-loop 4-point amplitude in (2.5). This suggests that the 6d 4-point amplitude is dual to a Wilson surface and can be described to all-loop orders in terms of a BDS-like formula. A novel feature of the on-shell action for the 2-brane compared to the on-shell action of the string obtained in [31] is an overall prefactor which diverges as $\epsilon \rightarrow 0$. A similar divergence was found in previous holographic calculations of Wilson surfaces of the $6 \mathrm{~d}(2,0)$ theory [65] and circular Wilson loops of 5d SYM [66], and can be associated with the conformal anomaly of Wilson surfaces [67-70]. Furthermore, it may be possible to interpret the prefactor in (3.18) which goes as $N / \epsilon$ as an t'Hooft coupling in the $6-2 \epsilon$ dimensions. We shall discuss this point further in section 4 .

\section{Discussion}

In this note, we have explored dual conformal symmetry for loop amplitudes in six dimensions. This study is motivated by the fact that the scattering amplitudes of D3 and M2-brane worldvolume theories have dual conformal symmetry even though it is hidden

\footnotetext{
${ }^{6}$ Note that $\tilde{\mathcal{S}}$ is identical to the regulated on-shell action for the string solution obtained in [31] apart from an additive constant. In [31], this constant was reported to be $1 / 2$ instead of $3 / 2$. For more details, see appendix B.
} 
from the point of view of their Lagrangian formulations. This suggests that it may also play a role in the scattering amplitudes of M5-brane worldvolume theories.

If we consider a hypothetical $6 \mathrm{~d}$ theory which has a planar limit and scattering amplitudes with rational (field theoretic) loop integrands, we find that imposing dual conformal symmetry fixes the integrand for the 4-point 1-loop amplitude. After performing the integral over loop momentum in $d=6-2 \epsilon$ dimensions, we obtain a result that is remarkably similar to the 4-point amplitude of $\mathcal{N}=4 \mathrm{SYM}$ and the ABJM theory. We also analyze two-loop 4-point amplitudes in six dimensions and identify two integrands compatible with dual conformal symmetry and unitarity.

Given that the planar scattering amplitudes in $\mathcal{N}=4 \mathrm{SYM}$ can be computed at strong from the area of a string worldsheet in AdS whose boundary corresponds to a null-polygonal Wilson loop, one may expect that the amplitudes of the $6 \mathrm{~d}$ theory can also be computed holographically by minimizing the volume of a 2-brane whose boundary corresponds to a Wilson surface which somehow encodes a null polygon. In fact, we find a simple generalization of the 4-cusp Alday-Maldacena solution which minimizes the action of a 2-brane in AdS and verify that its on-shell action has essentially the same structure as the 1-loop 4-point amplitude we deduced using dual conformal symmetry. This suggests that the $6 \mathrm{~d}$ 4-point amplitude is dual to a Wilson surface and can be described to all-loop orders using a formula analogous to the BDS formula of $\mathcal{N}=4$ SYM. A new feature of the on-shell action for the 2-brane compared to that of the string in AdS is an overall prefactor which diverges as $\epsilon \rightarrow 0$. This additional divergence was observed in previous holographic calculations of Wilson surfaces.

Although our calculations are highly suggestive, the existence of a $6 \mathrm{~d}$ theory with these properties and its relation to M5 branes is still rather speculative. In particular, there are a number of questions that deserve further study:

- Perhaps the most immediate task would be to extend our perturbative 4-point calculations to higher loops and check if they are consistent with an all-loop formula for the 4-point amplitude. This would require extending our 1-loop calculation to $\mathcal{O}\left(\epsilon^{2}\right)$, computing the 2-loop Mellin-Barnes integrals we obtained in appendix A, and determining if any linear combination of these integrals can arise from exponentiating the 1-loop amplitude. In the end, it may only be necessary to consider the two integrands we describe in section 2.4, whose Mellin-Barnes representations are very similar to that of the 2-loop 4-point amplitude of $\mathcal{N}=4$ SYM.

- Note that two of the propagators in the 1-loop integrand in (2.3) are squared, which suggests that if there is an underlying Lagrangian, it contains terms of the form $\phi \partial^{4} \phi$. Such theories are not generally unitary unless they arise from expansions of non-local theories like string theory [80]. This is certainly a possibility for the M5-brane worldvolume theory given that it has been argued to be a non-gravitational self-dual string theory [81, 82]. Another possibility is that the $6 \mathrm{~d}$ theory in question is not unitary. Indeed, four-derivative terms appear in certain non-unitary $6 \mathrm{~d}$ superconformal theories $[74,75]$, so studying the scattering amplitudes of these theories is an important direction for future research. In [61], it was proven that it is not possible to construct 
tree-level amplitudes of self-dual tensor multiplets assuming unitarity and locality. It would be interesting to revisit this question in light of our loop-level calculations, which suggest that some of these assumptions may need to be relaxed.

- A crucial assumption in our analysis is the existence of a planar limit. In order to define such a limit, there must be a coupling which can be sent to zero as the rank of the gauge group $N$ is sent to infinity. For the M2-brane worldvolume theory, this can be accomplished by performing a $\mathbb{Z}_{k}$ orbifold of the space transverse to the M2branes, which ultimately gives rise to the 't Hooft coupling $N / k$. For the M5-brane worldvolume theory, our results suggest that the coupling is tied to the dimension of the worldvolume itself. In particular, note that the prefactor of the on-shell action in (3.18) is proportional to $N / \epsilon$. If we interpret this as the 't Hooft coupling, we see that the coupling diverges as $d \rightarrow 6$. This is reminiscent of the conjecture that $5 \mathrm{~d}$ SYM is equivalent to the $6 \mathrm{~d}(2,0)$ compactified on a circle $[22,23]$, where the radius of compactification is proportional to the $5 \mathrm{~d}$ coupling [83]. It would therefore be interesting to explore how our results relate to the planar scattering amplitudes and Wilson loops of 5d SYM. Although perturbative amplitudes of 5d SYM have UV divergences [84], it has been argued that they may be removed by non-perturbative effects [85].

- In $\mathcal{N}=4 \mathrm{SYM}$, dual superconformal symmetry was shown to arise from self-duality of the dual string theory under a combination of bosonic and fermionic T-duality transformations. It would therefore be interesting to look for an analogue of Tduality in M-theory, perhaps by compactifying the theory on a circle to obtain IIA string theory, performing various combinations of bosonic and fermionic T-duality transformations, and then decompactifying back to M-theory. Some of these ideas were considered in [86].

Ultimately, we hope this line of investigation will yield new insight into the longstanding question of how to formulate the worldvolume theory of M5-branes.

\section{Acknowledgments}

We thank Paul Heslop, Simon Ross, Douglas Smith, and Tadashi Takayanagi for helpful comments on the manuscript. JB is supported by the STFC Consolidated Grant ST/L000407/1. AL is supported by the Royal Society as a Royal Society University Research Fellowship holder.

\section{A Details of 2-loop 4-point amplitude}

In general, the 2-loop integrand with a double box topology has the following form:

$$
\mathcal{A}_{4}^{(2)}=\int d^{6-2 \epsilon} x_{0} d^{6-2 \epsilon} x_{5} \frac{\left(x_{13}^{2}\right)^{\alpha_{1}}\left(x_{24}^{2}\right)^{\alpha_{2}}}{\left(x_{01}^{2}\right)^{\nu_{2}}\left(x_{02}^{2}\right)^{\nu_{1}}\left(x_{04}^{2}\right)^{\nu_{3}}\left(x_{52}^{2}\right)^{\nu_{6}}\left(x_{53}^{2}\right)^{\nu_{5}}\left(x_{54}^{2}\right)^{\nu_{4}}\left(x_{05}^{2}\right)^{\nu_{7}}}+s \leftrightarrow t .
$$




\begin{tabular}{|c|c|c|c|c|c|c|c|c|c|}
\hline No. & $\nu_{1}$ & $\nu_{2}$ & $\nu_{3}$ & $\nu_{4}$ & $\nu_{5}$ & $\nu_{6}$ & $\nu_{7}$ & $\alpha_{1}$ & $\alpha_{2}$ \\
\hline$(1)$ & 1 & 2 & 1 & 1 & 2 & 1 & 2 & 2 & 2 \\
$(2)$ & 2 & 1 & 2 & 2 & 1 & 2 & 1 & 1 & 4 \\
$(3)$ & 1 & 1 & 1 & 1 & 1 & 1 & 3 & 1 & 2 \\
$(4)$ & 2 & 1 & 1 & 2 & 1 & 1 & 2 & 1 & 3 \\
$(5)$ & 1 & 1 & 2 & 1 & 1 & 2 & 2 & 1 & 3 \\
$(6)$ & 3 & 1 & 1 & 3 & 1 & 1 & 1 & 1 & 4 \\
$(7)$ & 1 & 1 & 3 & 1 & 1 & 3 & 1 & 1 & 4 \\
$(8)$ & 1 & 2 & 2 & 1 & 2 & 2 & 1 & 2 & 3 \\
$(9)$ & 2 & 2 & 1 & 2 & 2 & 1 & 1 & 2 & 3 \\
$(10)$ & 1 & 3 & 1 & 1 & 3 & 1 & 1 & 3 & 2 \\
\hline
\end{tabular}

Table 1. Table listing sets of exponents in (A.1) consistent with dual conformal symmetry.

There are ten possibilities consistent with dual conformal symmetry, which we summarize in table 1. The integral in (A.1) has a Mellin-Barnes representation

$$
\begin{aligned}
\mathcal{I}\left(\nu_{1}, \nu_{2}, \nu_{3}, \nu_{4}, \nu_{5}, \nu_{6}, \nu_{7}, \alpha_{1}, \alpha_{2}\right) & \\
= & s^{\alpha_{1}+D-\nu} t^{\alpha_{2}} \frac{(-1)^{D+1} \pi^{D}}{\Gamma\left(D-\nu_{4567}\right) \prod_{l=2,4,5,6,7} \Gamma\left(\nu_{l}\right)} \int \prod_{j=1}^{4} \frac{d z_{j}}{2 \pi i}\left(\frac{t}{s}\right)^{z_{1}} \Gamma\left(\nu_{2}+z_{1}\right) \Gamma\left(-z_{1}\right) \\
& \times \frac{\Gamma\left(z_{2}+z_{4}\right) \Gamma\left(z_{3}+z_{4}\right) \Gamma\left(\nu_{123}-D / 2+z_{4}\right) \Gamma\left(\nu_{7}+z_{1}-z_{4}\right)}{\Gamma\left(\nu_{1} z_{3}+z_{4}\right) \Gamma\left(\nu_{3}+z_{2}+z_{4}\right) \Gamma\left(D-\nu_{123}+z_{1}-z_{4}\right)} \Gamma\left(-z_{2}-z_{3}-z_{4}\right) \\
& \times \Gamma\left(\nu_{5}+z_{1}+z_{2}+z_{3}+z_{4}\right) \Gamma\left(\nu_{4567}-D / 2+z_{1}-z_{4}\right) \Gamma\left(D / 2-\nu_{12}+z_{2}\right) \\
& \times \Gamma\left(D / 2-\nu 23+z_{3}\right) \Gamma\left(D / 2-\nu_{567}-z_{1}-z_{2}\right) \Gamma\left(D / 2-\nu 457-z_{1}-z_{3}\right),
\end{aligned}
$$

where $D=6-2 \epsilon, \nu=\sum_{i=1}^{7} \nu_{i}, \nu_{i j}=\nu_{i}+\nu_{j}$, and $\nu_{i j k}=\nu_{i}+\nu_{j}+\nu_{k}$. Note that first and the second sets of exponents listed in table 1 correspond to the integrals (2.6), and we shall denote their Mellin Barnes integrands as $I_{(1)}^{6 d}$ and $I_{(2)}^{6 d}$, respectively. For comparison, the Mellin-Barnes representation of the planar 4-point 2-loop amplitude in $\mathcal{N}=4 \mathrm{SYM}$ is given by $\mathcal{I}(1,1,1,1,1,1,1,1,0)$ and we will denote the Mellin-Barnes integrand as $I^{4 d}$. Remarkably, the Mellin-Barnes integrands corresponding to (2.6) are very similar to that of $\mathcal{N}=4$ SYM:

$$
\begin{aligned}
\frac{I^{4 d}\left(z_{1}, z_{2}, z_{3}, z_{4}\right)}{I_{(1)}^{6 d}\left(z_{1}-1, z_{2}, z_{3}, z_{4}\right)} & =-\frac{t \Gamma\left(-z_{1}\right)}{\pi^{2} \Gamma\left(1-z_{1}\right)} \\
\frac{I^{4 d}\left(z_{1}, z_{2}, z_{3}, z_{4}\right)}{I_{(2)}^{6 d}\left(z_{1}, z_{2}, z_{3}, z_{4}\right)} & =\frac{s^{2} \Gamma\left(z_{2}+z_{4}+2\right) \Gamma\left(z_{3}+z_{4}+2\right) \Gamma\left(z_{4}+\epsilon+1\right) \Gamma\left(z_{1}-z_{4}+\epsilon+2\right)}{\pi^{2} \Gamma\left(z_{2}+z_{4}+1\right) \Gamma\left(z_{3}+z_{4}+1\right) \Gamma\left(z_{4}+\epsilon+2\right) \Gamma\left(z_{1}-z_{4}+\epsilon+3\right)} .
\end{aligned}
$$




\section{B Details of regularized action}

The regulated on-shell action in (3.17) takes the form ${ }^{7}$

$$
i S=-T_{\epsilon} \int_{-\infty}^{+\infty} d \xi_{1} d \xi_{2} \frac{1+\epsilon I_{1}+\epsilon^{2}\left(I_{2}-2 I_{2}^{2}+\frac{1}{4}\right)}{\left(\cosh \xi_{1} \cosh \xi_{2}+b \sinh \xi_{1} \sinh \xi_{2}\right)^{\epsilon}}
$$

where

$$
\begin{aligned}
& I_{1}=\frac{\left(b^{2}-1\right) \cosh \left(2 \xi_{1}\right)+\left(b^{2}-1\right) \cosh \left(2 \xi_{2}\right)-2\left(b^{2}+1\right)}{8\left(b \sinh \left(\xi_{1}\right) \sinh \left(\xi_{2}\right)+\cosh \left(\xi_{1}\right) \cosh \left(\xi_{2}\right)\right)^{2}} \\
& I_{2}=\frac{-\left(b^{2}+1\right) \cosh \left(2 \xi_{1}\right) \cosh \left(2 \xi_{2}\right)-2 b \sinh \left(2 \xi_{1}\right) \sinh \left(2 \xi_{2}\right)+1+b^{2}}{16\left(b \sinh \left(\xi_{1}\right) \sinh \left(\xi_{2}\right)+\cosh \left(\xi_{1}\right) \cosh \left(\xi_{2}\right)\right)^{2}} \\
& \mathcal{T}_{\epsilon}=R^{3} \mathcal{T}_{M 2} \int \frac{d \xi_{3}}{\left(1-\xi_{3}^{2}\right)^{2+\frac{\epsilon}{2}}}=R^{3} \mathcal{T}_{M_{2}} \frac{\sqrt{\pi} \Gamma\left(-1-\frac{\epsilon}{2}\right)}{\Gamma\left(-\frac{1}{2}-\frac{\epsilon}{2}\right)}
\end{aligned}
$$

The integrals evaluate to

$$
\begin{aligned}
\int_{-\infty}^{+\infty} d \xi_{1} d \xi_{2} \frac{1}{\left(\cosh \xi_{1} \cosh \xi_{2}+b \sinh \xi_{1} \sinh \xi_{2}\right)^{\epsilon}} & =\frac{\pi \Gamma\left(-\frac{\epsilon}{2}\right)^{2}}{\Gamma\left(\frac{1-\epsilon}{2}\right)^{2}} F_{1}\left(\frac{1}{2},-\frac{\epsilon}{2} ; \frac{1}{2}-\frac{\epsilon}{2} ; b^{2}\right) \\
\int_{-\infty}^{+\infty} d \xi_{1} d \xi_{2} \frac{I_{1}}{\left(\cosh \xi_{1} \cosh \xi_{2}+b \sinh \xi_{1} \sinh \xi_{2}\right)^{\epsilon}} & =\frac{2}{\epsilon}+\mathcal{O}\left(\epsilon^{0}\right) \\
\int_{-\infty}^{+\infty} d \xi_{1} d \xi_{2} \frac{I_{2}-2 I_{2}^{2}+\frac{1}{4}}{\left(\cosh \xi_{1} \cosh \xi_{2}+b \sinh \xi_{1} \sinh \xi_{2}\right)^{\epsilon}} & =-\frac{1}{2 \epsilon^{2}}+\mathcal{O}\left(\frac{1}{\epsilon}\right) .
\end{aligned}
$$

Using the following expansions

$$
\begin{aligned}
\frac{\pi \Gamma\left(-\frac{\epsilon}{2}\right)^{2}}{\Gamma\left(\frac{1-\epsilon}{2}\right)^{2}} & =\frac{4}{\epsilon^{2}}-\frac{4 \log (4)}{\epsilon}+\left(2 \log ^{2}(4)-\frac{\pi^{2}}{3}\right)+\mathcal{O}(\epsilon) \\
{ }_{2} F_{1}\left(\frac{1}{2},-\frac{\epsilon}{2} ; \frac{1}{2}-\frac{\epsilon}{2} ; b^{2}\right) & =\left(\frac{1}{2}(1-b)^{\epsilon}+\frac{1}{2}(1-b)^{\epsilon}\right)-\frac{\epsilon^{2}}{4} \ln \left(\frac{1+b}{1-b}\right)+\mathcal{O}\left(\epsilon^{3}\right) \\
T_{\epsilon} & =-\frac{R^{3} \mathcal{T}_{M 2}}{\epsilon}+\mathcal{O}\left(\epsilon^{0}\right)
\end{aligned}
$$

and putting all the terms together gives (3.18).

Open Access. This article is distributed under the terms of the Creative Commons Attribution License (CC-BY 4.0), which permits any use, distribution and reproduction in any medium, provided the original author(s) and source are credited.

\footnotetext{
${ }^{7}$ Note that on evaluating the onshell Nambu-Goto action, in the $\mathcal{O}(\epsilon)^{2}$ term, we find an extra additive $\frac{1}{4}$, compared to [31]. This eventually contributes to the constant $\mathcal{O}(\epsilon)^{0}$ term, in the amplitude. We think this should be present for the case of the string worldsheet in [31] as well.
} 


\section{References}

[1] J.M. Maldacena, The Large-N limit of superconformal field theories and supergravity, Int. J. Theor. Phys. 38 (1999) 1113 [hep-th/9711200] [INSPIRE].

[2] M.B. Green and J.H. Schwarz, Supersymmetrical String Theories, Phys. Lett. B 109 (1982) 444 [INSPIRE].

[3] P.G.O. Freund and M.A. Rubin, Dynamics of Dimensional Reduction, Phys. Lett. B 97 (1980) 233 [INSPIRE].

[4] K. Pilch, P. van Nieuwenhuizen and P.K. Townsend, Compactification of $d=11$ Supergravity on $S_{4}($ Or $11=7+4$, Too), Nucl. Phys. B 242 (1984) 377 [inSPIRE].

[5] L. Brink, J.H. Schwarz and J. Scherk, Supersymmetric Yang-Mills Theories, Nucl. Phys. B 121 (1977) 77 [INSPIRE].

[6] G. 't Hooft, A Planar Diagram Theory for Strong Interactions, Nucl. Phys. B 72 (1974) 461 [INSPIRE].

[7] N. Beisert et al., Review of AdS/CFT Integrability: An Overview, Lett. Math. Phys. 99 (2012) 3 [arXiv: 1012.3982] [INSPIRE].

[8] O. Aharony, O. Bergman, D.L. Jafferis and J. Maldacena, $N=6$ superconformal Chern-Simons-matter theories, M2-branes and their gravity duals, JHEP 10 (2008) 091 [arXiv:0806.1218] [INSPIRE].

[9] A. Gustavsson, Algebraic structures on parallel M2-branes, Nucl. Phys. B 811 (2009) 66 [arXiv:0709.1260] [INSPIRE].

[10] J. Bagger and N. Lambert, Gauge symmetry and supersymmetry of multiple M2-branes, Phys. Rev. D 77 (2008) 065008 [arXiv:0711.0955] [INSPIRE].

[11] J. Bagger and N. Lambert, Comments on multiple M2-branes, JHEP 02 (2008) 105 [arXiv:0712.3738] [INSPIRE].

[12] D. Gaiotto, $N=2$ dualities, JHEP 08 (2012) 034 [arXiv:0904.2715] [INSPIRE].

[13] L.F. Alday, D. Gaiotto and Y. Tachikawa, Liouville Correlation Functions from Four-dimensional Gauge Theories, Lett. Math. Phys. 91 (2010) 167 [arXiv:0906.3219] [INSPIRE].

[14] T. Dimofte, D. Gaiotto and S. Gukov, Gauge Theories Labelled by Three-Manifolds, Commun. Math. Phys. 325 (2014) 367 [arXiv:1108.4389] [InSPIRE].

[15] M. Perry and J.H. Schwarz, Interacting chiral gauge fields in six-dimensions and Born-Infeld theory, Nucl. Phys. B 489 (1997) 47 [hep-th/9611065] [INSPIRE].

[16] P. Pasti, D.P. Sorokin and M. Tonin, Covariant action for a $D=11$ five-brane with the chiral field, Phys. Lett. B 398 (1997) 41 [hep-th/9701037] [INSPIRE].

[17] O. Aharony, M. Berkooz, S. Kachru, N. Seiberg and E. Silverstein, Matrix description of interacting theories in six-dimensions, Adv. Theor. Math. Phys. 1 (1998) 148 [hep-th/9707079] [INSPIRE].

[18] N. Arkani-Hamed, A.G. Cohen, D.B. Kaplan, A. Karch and L. Motl, Deconstructing $(2,0)$ and little string theories, JHEP 01 (2003) 083 [hep-th/0110146] [INSPIRE].

[19] N. Lambert and C. Papageorgakis, Nonabelian (2,0) Tensor Multiplets and 3-algebras, JHEP 08 (2010) 083 [arXiv: 1007.2982] [INSPIRE]. 
[20] C. Sämann and M. Wolf, Non-Abelian Tensor Multiplet Equations from Twistor Space, Commun. Math. Phys. 328 (2014) 527 [arXiv:1205.3108] [InSPIRE].

[21] H. Samtleben, E. Sezgin and R. Wimmer, Six-dimensional superconformal couplings of non-abelian tensor and hypermultiplets, JHEP 03 (2013) 068 [arXiv:1212.5199] [INSPIRE].

[22] M.R. Douglas, On D = 5 super Yang-Mills theory and (2,0) theory, JHEP 02 (2011) 011 [arXiv: 1012.2880] [INSPIRE].

[23] N. Lambert, C. Papageorgakis and M. Schmidt-Sommerfeld, M5-Branes, D4-branes and Quantum 5D super-Yang-Mills, JHEP 01 (2011) 083 [arXiv:1012.2882] [INSPIRE].

[24] A.E. Lipstein and R.A. Reid-Edwards, Lattice Gerbe Theory, JHEP 09 (2014) 034 [arXiv: 1404.2634] [INSPIRE].

[25] J.M. Drummond, J. Henn, V.A. Smirnov and E. Sokatchev, Magic identities for conformal four-point integrals, JHEP 01 (2007) 064 [hep-th/0607160] [INSPIRE].

[26] A. Brandhuber, P. Heslop and G. Travaglini, A Note on dual superconformal symmetry of the $N=4$ super Yang-Mills S-matrix, Phys. Rev. D 78 (2008) 125005 [arXiv:0807.4097] [INSPIRE].

[27] J.M. Drummond, J. Henn, G.P. Korchemsky and E. Sokatchev, Dual superconformal symmetry of scattering amplitudes in $N=4$ super-Yang-Mills theory, Nucl. Phys. B 828 (2010) 317 [arXiv:0807.1095] [INSPIRE].

[28] T. Bargheer, F. Loebbert and C. Meneghelli, Symmetries of Tree-level Scattering Amplitudes in $N=6$ Superconformal Chern-Simons Theory, Phys. Rev. D 82 (2010) 045016 [arXiv: 1003.6120] [INSPIRE].

[29] Y.-t. Huang and A.E. Lipstein, Dual Superconformal Symmetry of $N=6$ Chern-Simons Theory, JHEP 11 (2010) 076 [arXiv: 1008.0041] [INSPIRE].

[30] D. Gang, Y.-t. Huang, E. Koh, S. Lee and A.E. Lipstein, Tree-level Recursion Relation and Dual Superconformal Symmetry of the ABJM Theory, JHEP 03 (2011) 116 [arXiv: 1012.5032] [INSPIRE].

[31] L.F. Alday and J.M. Maldacena, Gluon scattering amplitudes at strong coupling, JHEP 06 (2007) 064 [arXiv: 0705.0303] [INSPIRE].

[32] A. Brandhuber, P. Heslop and G. Travaglini, $M H V$ amplitudes in $N=4$ super Yang-Mills and Wilson loops, Nucl. Phys. B 794 (2008) 231 [arXiv:0707.1153] [inSPIRE].

[33] J.M. Drummond, J. Henn, G.P. Korchemsky and E. Sokatchev, On planar gluon amplitudes/Wilson loops duality, Nucl. Phys. B 795 (2008) 52 [arXiv:0709.2368] [INSPIRE].

[34] J.M. Drummond, G.P. Korchemsky and E. Sokatchev, Conformal properties of four-gluon planar amplitudes and Wilson loops, Nucl. Phys. B 795 (2008) 385 [arXiv:0707.0243] [INSPIRE].

[35] L.J. Mason and D. Skinner, The Complete Planar S-matrix of $N=4$ SYM as a Wilson Loop in Twistor Space, JHEP 12 (2010) 018 [arXiv: 1009.2225] [INSPIRE].

[36] S. Caron-Huot, Notes on the scattering amplitude/Wilson loop duality, JHEP 07 (2011) 058 [arXiv: 1010.1167] [INSPIRE].

[37] N. Berkovits and J. Maldacena, Fermionic T-duality, Dual Superconformal Symmetry and the Amplitude/Wilson Loop Connection, JHEP 09 (2008) 062 [arXiv:0807.3196] [INSPIRE]. 
[38] N. Beisert, R. Ricci, A.A. Tseytlin and M. Wolf, Dual Superconformal Symmetry from $A d S_{5} \times S^{5}$ Superstring Integrability, Phys. Rev. D 78 (2008) 126004 [arXiv:0807.3228] [INSPIRE].

[39] I. Adam, A. Dekel and Y. Oz, On the fermionic T-duality of the $A d S_{4} \times C P^{3} \sigma$-model, JHEP 10 (2010) 110 [arXiv: 1008.0649] [INSPIRE].

[40] I. Bakhmatov, On $A d S_{4} \times C P^{3} T$-duality, Nucl. Phys. B 847 (2011) 38 [arXiv:1011.0985] [INSPIRE].

[41] E.Ó. Colgáin and A. Pittelli, A Requiem for AdS $S_{4} \times C P^{3}$ Fermionic self-T-duality, Phys. Rev. D 94 (2016) 106006 [arXiv: 1609.03254] [INSPIRE].

[42] L. Dolan, C.R. Nappi and E. Witten, Yangian symmetry in $D=4$ superconformal Yang-Mills theory, in proceedings of the 3rd International Symposium on Quantum theory and symmetries (QTS3), Cincinnati, U.S.A., 10-14 Sept. 2003, pp. 300-315 [hep-th/0401243] [INSPIRE].

[43] J.M. Drummond, J.M. Henn and J. Plefka, Yangian symmetry of scattering amplitudes in $N=4$ super Yang-Mills theory, JHEP 05 (2009) 046 [arXiv:0902.2987] [INSPIRE].

[44] Z. Bern, L.J. Dixon, D.C. Dunbar and D.A. Kosower, One-loop n-point gauge theory amplitudes, unitarity and collinear limits, Nucl. Phys. B 425 (1994) 217 [hep-ph/9403226] [INSPIRE].

[45] Z. Bern, L.J. Dixon, D.C. Dunbar and D.A. Kosower, Fusing gauge theory tree amplitudes into loop amplitudes, Nucl. Phys. B 435 (1995) 59 [hep-ph/9409265] [INSPIRE].

[46] R. Britto, F. Cachazo, B. Feng and E. Witten, Direct proof of tree-level recursion relation in Yang-Mills theory, Phys. Rev. Lett. 94 (2005) 181602 [hep-th/0501052] [INSPIRE].

[47] J.M. Drummond, J. Henn, G.P. Korchemsky and E. Sokatchev, Conformal Ward identities for Wilson loops and a test of the duality with gluon amplitudes, Nucl. Phys. B 826 (2010) 337 [arXiv:0712.1223] [INSPIRE].

[48] Z. Bern, L.J. Dixon and V.A. Smirnov, Iteration of planar amplitudes in maximally supersymmetric Yang-Mills theory at three loops and beyond, Phys. Rev. D 72 (2005) 085001 [hep-th/0505205] [INSPIRE].

[49] Z. Bern, M. Czakon, L.J. Dixon, D.A. Kosower and V.A. Smirnov, The Four-Loop Planar Amplitude and Cusp Anomalous Dimension in Maximally Supersymmetric Yang-Mills Theory, Phys. Rev. D 75 (2007) 085010 [hep-th/0610248] [INSPIRE].

[50] F. Cachazo, M. Spradlin and A. Volovich, Iterative structure within the five-particle two-loop amplitude, Phys. Rev. D 74 (2006) 045020 [hep-th/0602228] [INSPIRE].

[51] Z. Bern, L.J. Dixon, D.A. Kosower et al., The Two-Loop Six-Gluon MHV Amplitude in Maximally Supersymmetric Yang-Mills Theory, Phys. Rev. D 78 (2008) 045007 [arXiv: 0803.1465] [INSPIRE].

[52] L.F. Alday and J. Maldacena, Comments on gluon scattering amplitudes via AdS/CFT, JHEP 11 (2007) 068 [arXiv:0710.1060] [INSPIRE].

[53] L.F. Alday, D. Gaiotto and J. Maldacena, Thermodynamic Bubble Ansatz, JHEP 09 (2011) 032 [arXiv:0911.4708] [INSPIRE].

[54] J.M. Henn, J. Plefka and K. Wiegandt, Light-like polygonal Wilson loops in $3 d$ Chern-Simons and ABJM theory, JHEP 08 (2010) 032 [Erratum ibid. 11 (2011) 053] [arXiv: 1004.0226] [INSPIRE]. 
[55] W.-M. Chen and Y.-t. Huang, Dualities for Loop Amplitudes of $N=6$ Chern-Simons Matter Theory, JHEP 11 (2011) 057 [arXiv:1107.2710] [INSPIRE].

[56] M.S. Bianchi, M. Leoni, A. Mauri, S. Penati and A. Santambrogio, Scattering Amplitudes/Wilson Loop Duality In ABJM Theory, JHEP 01 (2012) 056 [arXiv:1107.3139] [INSPIRE].

[57] M.S. Bianchi, M. Leoni, A. Mauri, S. Penati and A. Santambrogio, Scattering in ABJ theories, JHEP 12 (2011) 073 [arXiv:1110.0738] [INSPIRE].

[58] M.S. Bianchi, M. Leoni and S. Penati, An All Order Identity between ABJM and $N=4$ SYM Four-Point Amplitudes, JHEP 04 (2012) 045 [arXiv:1112.3649] [INSPIRE].

[59] M.S. Bianchi, G. Giribet, M. Leoni and S. Penati, Light-like Wilson loops in ABJM and maximal transcendentality, JHEP 08 (2013) 111 [arXiv: 1304.6085] [INSPIRE].

[60] M.S. Bianchi and M. Leoni, On the ABJM four-point amplitude at three loops and BDS exponentiation, JHEP 11 (2014) 077 [arXiv: 1403.3398] [INSPIRE].

[61] Y.-t. Huang and A.E. Lipstein, Amplitudes of 3D and 6D Maximal Superconformal Theories in Supertwistor Space, JHEP 10 (2010) 007 [arXiv:1004.4735] [INSPIRE].

[62] T. Dennen and Y.-t. Huang, Dual Conformal Properties of Six-Dimensional Maximal Super Yang-Mills Amplitudes, JHEP 01 (2011) 140 [arXiv:1010.5874] [INSPIRE].

[63] S. Caron-Huot and D. O'Connell, Spinor Helicity and Dual Conformal Symmetry in Ten Dimensions, JHEP 08 (2011) 014 [arXiv:1010.5487] [INSPIRE].

[64] A.E. Lipstein and L. Mason, Amplitudes of 3d Yang-Mills Theory, JHEP 01 (2013) 009 [arXiv: 1207.6176] [INSPIRE].

[65] D.E. Berenstein, R. Corrado, W. Fischler and J.M. Maldacena, The Operator product expansion for Wilson loops and surfaces in the large-N limit, Phys. Rev. D 59 (1999) 105023 [hep-th/9809188] [INSPIRE].

[66] D. Young, Wilson Loops in Five-Dimensional super-Yang-Mills, JHEP 02 (2012) 052 [arXiv:1112.3309] [INSPIRE].

[67] C.R. Graham and E. Witten, Conformal anomaly of submanifold observables in AdS/CFT correspondence, Nucl. Phys. B 546 (1999) 52 [hep-th/9901021] [INSPIRE].

[68] M. Henningson and K. Skenderis, Weyl anomaly for Wilson surfaces, JHEP 06 (1999) 012 [hep-th/9905163] [INSPIRE].

[69] A. Gustavsson, On the Weyl anomaly of Wilson surfaces, JHEP 12 (2003) 059 [hep-th/0310037] [INSPIRE].

[70] A. Gustavsson, Conformal anomaly of Wilson surface observables: A Field theoretical computation, JHEP 07 (2004) 074 [hep-th/0404150] [INSPIRE].

[71] H. Mori and S. Yamaguchi, M5-branes and Wilson surfaces in $A d S_{7} / C F T_{6}$ correspondence, Phys. Rev. D 90 (2014) 026005 [arXiv: 1404.0930] [InSPIRE].

[72] R. Corrado, B. Florea and R. McNees, Correlation functions of operators and Wilson surfaces in the $D=6,(0,2)$ theory in the large- $N$ limit, Phys. Rev. D 60 (1999) 085011 [hep-th/9902153] [INSPIRE].

[73] L.J. Dixon, A brief introduction to modern amplitude methods, in proceedings of the 2012 European School of High-Energy Physics (ESHEP 2012), La Pommeraye, Anjou, France, 6-19 June 2012, pp. 31-67 [arXiv: 1310.5353] [INSPIRE]. 
[74] E.A. Ivanov, A.V. Smilga and B.M. Zupnik, Renormalizable supersymmetric gauge theory in six dimensions, Nucl. Phys. B 726 (2005) 131 [hep-th/0505082] [INSPIRE].

[75] M. Beccaria and A.A. Tseytlin, Conformal a-anomaly of some non-unitary $6 d$ superconformal theories, JHEP 09 (2015) 017 [arXiv: 1506.08727] [INSPIRE].

[76] V.A. Smirnov, Evaluating Feynman Integrals, Springer (2005).

[77] A.I. Davydychev and J.B. Tausk, A Magic connection between massive and massless diagrams, Phys. Rev. D 53 (1996) 7381 [hep-ph/9504431] [InSPIRE].

[78] E. Bergshoeff, E. Sezgin and P.K. Townsend, Supermembranes and Eleven-Dimensional Supergravity, Phys. Lett. B 189 (1987) 75 [InSPIRE].

[79] T. Ortín, A Note on the D2-brane of the massive type IIA theory and gauged $\sigma$-models, Phys. Lett. B 415 (1997) 39 [hep-th/9707113] [INSPIRE].

[80] J.Z. Simon, Higher Derivative Lagrangians, Nonlocality, Problems and Solutions, Phys. Rev. D 41 (1990) 3720 [INSPIRE].

[81] E. Witten, Five-branes and M-theory on an orbifold, Nucl. Phys. B 463 (1996) 383 [hep-th/9512219] [INSPIRE].

[82] A. Strominger, Open p-branes, Phys. Lett. B 383 (1996) 44 [hep-th/9512059] [InSPIRE].

[83] E. Witten, Geometric Langlands From Six Dimensions, arXiv:0905.2720 [INSPIRE].

[84] Z. Bern, J.J. Carrasco, L.J. Dixon, M.R. Douglas, M. von Hippel and H. Johansson, $D=5$ maximally supersymmetric Yang-Mills theory diverges at six loops, Phys. Rev. D 87 (2013) 025018 [arXiv: 1210.7709] [INSPIRE].

[85] C. Papageorgakis and A.B. Royston, Revisiting Soliton Contributions to Perturbative Amplitudes, JHEP 09 (2014) 128 [arXiv:1404.0016] [INSPIRE].

[86] I. Jeon, N. Lambert and P. Richmond, Periodic Arrays of M2-Branes, JHEP 11 (2012) 100 [arXiv:1206.6699] [INSPIRE]. 